\title{
REFLEXIONES EN TORNO AL FRACASO ESCOLAR
}

\author{
M.P.I. Maria Esther Morales * \\ M.Sc. Natalia Salas **
}

Este artículo presenta algunas reflexiones sobre un aspecto de vital trascendencia en el contexto educativo actual: el fracaso escolar.

Para ello se retoman ideas, conceptos y experiencias de diversas fuentes, construyendo, desde un horizonte teórico, algunas nociones involucradas activamente en esta problemática social.

Parafacilitar su lectura, se divide en varios apartados, en donde se retoman temas tales como conceptualización del fracaso escolar, magnitud del problema en Costa Rica, aspectos asociados y variables que se derivan de lo observado dentro del sistema educativo, familiar, social y propio del estudiante. Dichos temas formulan postulaciones reconocidas y a la vez innovadoras sobre la necesidad de visualizar la problemática desde una perspectiva multicausal y a la vez integral, proponiendo un trabajo interdisciplinario, de cara a un mundo educativo cambiante y lleno de nuevos retos.
This article analyzes a matter of vital importance in the current edicational context: school failure.

Some ideas, concepts, and experiences that are actively involved in this social problem are reviewed from different sources, and summarized, from a theoretical viewpoint.

This text is divided in sections that overview the main notions on school failure, magnitude of the problem in Costa Rica, associated aspects and variables derived from studies and observations among the educational and social system, and also through the family patterns and personal ways of dealing with $i$. This sections postulate innovative and traditional ways to visualize this problem from a holistic and multicausal perspective; proposing an interdisciplinary work to deal with an educational world full of changes and challenges.

* Máster en Psicología Industrial y Organizacional, por la Universidad Latina de Costa Rica; en Psicología por la Universidad de Costa Rica. Actualmente es académica y coordina el Programa "Apoyo Psicopedagogico a la Educación Formal y No Formal" en el Instituto de Estudios Interdisciplinarios de la Niñez y Adolescencia del CIDE, Uiversidad Nacional.

** Máster en Psicología Clínica por la Universidad de Iberoamérica; en Psicología por la Universidad de Costa Rica, y egresada de Licenciatura de la misma universidad. Actualmente es académica y coordina el Programa "Desarrollo de la Niñez y la Adolescencia" y participa en el programa "Apoyo Psicopedagógico a la Educación Formal y No Formal", en el Instituto de Estudios Interdisciplinarios de la Nin̄ez y la Adolescencia. 


\section{¿Qué es el fracaso escolar?}

Uno de los mayores problemas que enfrenta hoy el Sistema Educativo y que tiene implicaciones morales, emocionales, sociales y económicas para los involucrados en el proceso, es el fracaso escolar de una gran parte de los niños y niñas, especialmente en la Educación Primaria.

Para efectos del presente documento, el fracaso escolar debe entenderse como los índices de repitencia y deserción que anualmente se presentan en estos grupos de población y que los marca irreversiblemente, dada la experiencia traumática en que esta situación se constituye, la más de las veces, por errores que cometen tanto los padres como los docentes y hasta el mismo sistema educativo.

El abandono de la primaria forma parte de una secuencia que se inicia con el ingreso a la escuela y es el resultado de factores familiares e individuales, pero, fundamentalmente, del fracaso en alcanzar la meta de superar las pruebas que se imponen en los sucesivos grados escolares.

Al respecto, la Oficina Internacional de la Educación de la UNESCO (sin año), plantea que "existen suficientes evidencias que indican que los altos índices de repetición en los primeros grados de la escuela primaria pueden atribuirse en buena medida, a la baja calidad de la oferta educativa". Estas evidencias señalan que el problema se concentra en los primeros años de la educación primaria, en algunas regiones del país, más que en otras y en alumnos de determinados estratos sociales o grupos de población.

Si partimos de que el escolar, con bajo rendimiento académico, es un niño o niña cuyo desempeño y eficiencia diaria en la escuela y en ocasiones, en diversos aspectos de su vida, es muy inferior a lo que se podría esperar de su desarrollo cognoscitivo, es acertado el planteamiento de Pazos (1991: 4), en el sentido que una de las mayores dificultades enfrentadas por la escuela pública es "asegurar que los niños alcancen efectivamente adecuados niveles de logro en el dominio de los conocimientos y destrezas culturales fundamentales que les permitan desempeñarse en la vida".

Frente a ello Rodríguez, Arce, Solís y Vargas (1997), nos comentan que cada niño que no alcanza los niveles más altos de educación, está detenido en el camino de desarrollarsus posibilidades y capacidades; está limitado como fuente de riqueza y disminuido en su dignidad de ser humano. Cuando se analizan diferentes factores de riesgo que podrían explicar el fracaso escolar en la población estudiantil, puede deducirse fácilmente la estrecha relación que existe entre la repitencia y la deserción, tanto a nivel cuantitativo como cualitativo.

Además, Martínez (1997), refiere que - con harta frecuencia- son diagnosticados como con trastornos de aprendizaje, niños con importantes 
El fracaso escolar debe entenderse como los indices de repitencia y deserción que anualmente se presentan en estos grupos de población y que los marca irreversiblemente, dada la experiencia traumática en que esta situación se constituye, la más de las veces por errores que cometen tanto los padres como los docentes y hasta el mismo Sistema Educativo problemas visuales como miopía o hiperme-tropía, al igual que niños con deficiencias auditivas entre ellos los hipoacústicos, sufriendo concomitantemente los resulta-dos de estos malos diagnósticos, y que, por ende, se incluyen en las listas del fracaso escolar.

Por otro lado, percibimos la sensación de "impotencia" del maestro que se encuentra desarmado, culpabilizado frente al fracaso de su alumno y que le crea una mala imagen de sí mismo.

Esto tiene repercusiones profundas también sobre la personalidad global del niño inadaptado, principalmente sobre el sentimiento de su valor personal. Esta vivencia de desvalorización, sentida por el escolar, va a constituirse en un handicap mayor para su integración ulterior, en una sociedad que valora el éxito precoz y la inteligencia.

Hay dos tipos de enfoques teóricos o razones para explicar la inadaptación recíproca de la escuela y del niño:

a. Se refiere al hecho de que la escuela es inadaptada en tanto que es una institución social que no toma en cuenta las desigualdades socio-culturales y reproduce un modelo cultural dominante.

b. Considera que el niño es inadaptado a causa de las desigualdades "naturales", inherentes a su personalidad.

Pero, ¿constituye la repetición un problema? A nivel teórico, Massimo (1995), sostiene que se trata más bien de una solución a las dificultades de aprendizaje experimentadas por algunos alumnos, a quienes se ofrece la oportunidad de un suplemento de enseñanza a fin de alcanzar el nivel de rendimiento académico requerido para aprobar el grado, según los estándares adoptados por el maestro de acuerdo con el currículo nacional. Desde este punto de vista, la repetición puede mejorar la preparación y las calificaciones para enfrentar las exigencias del examen.

En esta perspectiva, los efectos de la repetición podrían considerarse positivos, ya que permitirían un mayor aprendizaje y un mejor rendimiento, ayudando a los alumnos a aprender, según el desarrollo físico e intelectual 
alcanzado. Sin embargo, el elevado número de alumnos que repiten el primer grado de la escuela primaria permite pensar que los problemas más relevantes no se sitúan tanto a nivel del aprendizaje, sino de la enseñanza. Además, no siempre existe una estricta correspondencia entre repetición y logros de aprendizaje.

Contrariamente a lo que se podría suponer, los repitentes, los que abandonan y los que tienen dificultades en el aprendizaje, no suelen recibir ninguna atención especial por parte del docente y por lo general, resultan ser menos atendidos.

\section{Magnitud del problema en Costa Rica}

El MEP, basándose en los criterios establecidos por la UNICEF, calcula la deserción como la razón entre la matrícula inicial y la matrícula final. Esto implica que durante el transcurso del ciclo lectivo, quedan excluidos los niños y niñas que terminan el curso, pero que no ingresan a grados superiores; y los niños y niñas que no ingresan del todo al sistema.

En un estudio realizado por la UNICEF, el MEP y el Instituto de Investigación para el Mejoramiento de la Calidad de la Educación Costarricense (IIMEC) de la Universidad de Costa Rica, en 1995, se determinó que existen en el año lectivo dos períodos críticos para la deserción escolar. El primero de ellos lo constituye el que va de mayo a junio; y el segundo, el que cuenta de octubre a noviembre, justo finalizando cada semestre y en coincidencia, con la entrega de notas o calificaciones.

En los datos arrojados para 1997, de acuerdo con Rodríguez y otros (1997) se presenta el primer grado como crítico, presentando un 4,88\% de deserción. Esto significa que, del total de estudiantes matriculados en primer grado en 1997 , cinco de cada 100 niños se han retirado.

En relación con la repitencia escolar, en el sistema educativo costarricense, se observa una importante coincidencia en su comportamiento, al comparársele con la deserción. Igualmente, conforme se avanza en los niveles escolares, los porcentajes muestran un descenso. En los tres primeros grados, se ubican la mayor cantidad de repitentes (I ciclo), siendo el primer grado, el que incluye el porcentaje más significativo.

Para el año de 1999, y según datos proporcionados por el Ministerio de Educación Pública (2000), durante el curso lectivo de 1999, desertaron en el I y II Ciclo del Sistema Educativo, un total de 23,368 estudiantes, de los cuales 440 pertenecen a escuelas privadas y 61 a semi-públicas; el resto corresponde a escuelas públicas. De este total, el $66 \%$ se ubica en las escuelas públicas de la zona rural del país, contra un $34 \%$, perteneciente a instituciones públicas urbanas. 
Con respecto al sexo de la población desertora, el $54 \%$ son hombres $(12,7069)$ y el $46 \%$ son mujeres $(10,662)$. Este patrón de predominio masculino en la población desertora, se manifiesta en los centros educativos urbanos y rurales en todo el país.

\section{Aspectos asociados al problema}

"La tasa de asistencia escolar es el porcentaje que asiste efectivamente sobre el total de personas de esa edad" (Sautu, 1995). Es una medida cuantitativa del funcionamiento del sistema educativo, mientras que la regularidad, el atraso y el abandono son medidas agregadas de su eficacia. Sin embargo, la gravedad del problema no reside en su magnitud, ni en lo cuantificable, sino en sus causas y consecuencias; es por eso, que el presente apartado realiza un análisis sobre aquellos aspectos o variables asociados al problema.

Al respecto, Brenes (1997) nos indica que cualquier tipo de patología o evento vital que interfiera con la forma en la que el niño percibe su entorno o en la expresión de esta percepción, puede interferir en el rendimiento escolar. La lista de patologías de origen neurológico, pediátrico, psiquiátrico o psicológico es enorme, ya que no nos podemos olvidar de la estrecha interacción que existe entre las funciones biológicas-psicológicas-espirituales con el medio y cómo el desequilibrio en cualquiera de estas esferas va a producir algún tipo de respuesta en las otras.

Los Manuales de Clasificación de Enfermedades Mentales son muy amplios en cuanto a la gran cantidad de patologías que afectan a los niños y que van a interferir con su rendimiento escolar. Estas patologías van desde retardo mental pasando por los rastornos producidos por la ansiedad (como fobias escolares, angustia de separación), el autismo en sus diferentes expresiones, hasta el déficit atencional con hiperactividad.

Las situaciones que llevan con más frecuencia a un niño al fracaso escolar, son multicausales y están relacionadas con las diferentes formas de situaciones intrafamiliares (perspectivas de desarrollo físico, psicológico y social), así como los distintos aspectos propios de nuestra cultura educativa, donde olvidamos que los niños son sujetos de derechos y donde somos los adultos los que tomamos las decisiones por ellos.

Para Sautu (1995, p. 47) "sin descartar las diferencias individuales, existen factores socio-económicos y culturales que dificultan la adaptación a la escuela; por ello es falaz aceptar una interpretación que ubica la causa en los individuos cuando el rendimiento es la consecuencia de esos factores sociales que incrementan o disminuyen las posibilidades de acceder a la escuela, permanecer en ella y concluirla satisfactoriamente". 


\section{Algunas variables asociadas al fracaso escolar}

Para Rodríguez y otros (1997), dicho fenómeno es multicausal, y en Costa Rica se determinan tres grandes áreas, relacionadas con el fracaso escolar:

\section{Variables propias del sistema}

a. Rotación del personal docente: un niño promedio cuyo maestro es interino, tiene 2.5 más veces el riesgo de repetir el año, en relación con un niño promedio, cuyo maestro está en propiedad.

b. Formación profesional del docente: un docente con la preparación académica adecuada, debe garantizar una práctica pedagógica más científica, lo que necesariamente contribuye a facilitar un aprendizaje más efectivo y significativo. Según el IIMEC (1993), un niño promedio cuyo maestro es aspirante, tiene cuatro veces más riesgo de repetir año, al compararlo con un niño promedio cuyo maestro no es aspirante. De acuerdo con esto, si se logra que el maestro tenga una mayor categoría profesional (superior a aspirante), se evitará que dos de cada diez niños repitan el año escolar

c. Relación profesor - alumno: entre más democrática y afectiva es la conducta del maestro, incluyendo su capacidad verbal y numérica y su satisfacción laboral, mayor es el rendimiento que se observa en los alumnos. Lamentablemente, muchos de los maestros que trabajan con poblaciones de alto riesgo social, tienden a reforzar el fracaso escolar a través de la indiferencia y la estigmatización, apoyados en un estilo de relación vertical y autoritaria.

d. Métodos de enseñanza: Bogantes y Ramírez (1993), afirman que un factor negativo son las metodologías equivocadas, los contenidos aburridos o repetidos, la carencia de motivación, la falta de interés y atención de los docentes y de la institución por la situación de los alumnos. La presentación del material de aprendizaje en forma poco atractiva y con contenidos sin significado personal, imponen barreras para el surgimiento de la necesidad de estudiar y de aprender.

e. Procesos administrativos: la falta de flexibilidad ante el cambio y la innovación, que ocurre en muchas de nuestras institucioneseducativas, tiene que ver con la satisfacción laboral del docente y su participación activa en la toma de decisiones.

f. Tamaño de la escuela: estudios realizados por el IIMEC, 1995; determinan que existe una relación directa entre el tamaño de la escuela y el rendimiento de sus estudiantes. Un niño promedio en una escuela con matrícula superior 
a los 500 niños, tiene $60 \%$ más riesgo de repetir el grado escolar, comparado con un niño matriculado en otro tipo de escuela, por la oportunidad del segundo de recibir atención personalizada.

\section{Variables familiares y sociales:}

a. Edad de los padres: se ha determinado que un niño promedio cuyo encargado o tutor es menor de 20 años, tiene 2.3 veces más el riesgo de repetir el grado, en comparación con un niño promedio cuyos tutores son mayores de esa edad.

b. Escolaridad de los padres: el factor de más riesgo es el analfabetismo. Se ha demostrado que el nivel de escolaridad de los padres está correlacionado a la mayor probabilidad de éxito escolar de los niños y las niñas.

c. Interés y motivación de la familia: existe una gran necesidad de que los padres de familia se involucren en los procesos de aprendizaje de sus hijos. El interés de los padres por las tareas de índole escolar es una situación que incrementa las probabilidades de éxito. Aello se suman, las manifestaciones de orgullo que muestran sus padres y la confianza en la responsabilidad de sus hijos en relación con las tareas escolares.

d. Ubicación geográfica: los niños y las niñas de área rural dispersa, tienen aproximadámente cuatro veces más riesgo de desertar de la escuela, comparado con los niños de áreas urbanas.

e. Tamaño de la familia: los niños provenientes de familias numerosas, tienen un mayor riesgo también de repetir los grados o incluso de desertar de la escuela; y además cuando en la familia existen antecedentes de trabajo infantil, estas probabilidades tienden a aumentar.

f. Relación familia-docente: el no establecimiento de una relación adecuada entre ambos, incide necesariamente en el fracaso escolar.

g. Situación económica de pobreza o extrema pobreza: independientemente de las causas, estos estudios han coincidido en que los estudiantes de estratos socioeconómicos más bajos constituyen casi la totalidad de los desertores. Es claro que un niño con problemas de alimentación no puede tener el mismo rendimiento cognoscitivo que un niño bien alimentado. La disponibilidad de materiales de apoyo didáctico adicionales es otro elemento por considerar.

h. Factores culturales: la sensación aprendida de que es "imposible" salir de la pobreza y el conformismo generado por ello, influye negativamente en la valoración que se pueda hacer de la educación como medio de ascenso social. 
i. El trabajo infantil: a criterio de Defensa del Niño Internacional (DNI, 2001), en 1998 , se tiene que 147,087 de la población infantil costarricene entre 5 y 17 años de edad se encontraba trabajando. De este porcentaje, un $70 \%$ eran varones y $30 \%$ mujeres; así como un $76 \%$ era de zona rural y un $24 \%$ de zona urbana. En total 15,4\% combinaba el trabajo con el estudio. De igual forma, el área de Niñez y Adolescencia de la Defensoría de los Habitantes, refiere que el $45 \%$ de los trabajadores șon menores de 15 años, y tienen un promedio de más de 26 horas laborales por semana, lo cual consituye el $7 \%$ del Producto Interno Bruto del país.

j. Ambiente familiar: la familia, como contexto primario del desarrollo socioafectivo, debe procurar la contención necesaria para el desarrollo armonioso del escolar. Sin embargo, encontramos niños y niñas que pertenecen a familias donde se propician estilos de comunicación caracterizados por el engaño, el reproche, la ridiculización y otros tipos de agresión.

\section{Variables prupias del estudiante}

a. Concepto de sí mismo: incide en el éxito o fracaso escolar, especialmente por las atribuciones que el niño se hace de sí mismo. Es fundamental la devolución afectiva que el maestro debe hacer, considerando siempre la relación entre el éxito obtenido y las habilidades del niño.

b. Niños con necesidades educativas especiales: es importante señalar que las dificultades que el niño presenta para aprender no constituyen en sí mismas, un factor de riesgo para la repitencia o deserción escolar. El riesgo está más bien asociado a la falta de sensibilidad institucional y a la falta de instrumentalización que permita brindar a estos niños la atención adecuada.

c. La motivación por el estudio y el aprendizaje: debe existir la motivación que le permita desarrollar estrategias para aprender y conocer el entorno y que a su vez le permitan descubrir soluciones de los problemas que a diario tiene que enfrentar en la cotidianidad de la vida. Por eso, el proceso de aprendizaje debe ser placentero, emotivo, retador, pues de lo contrario la poca necesidad que pudiera existir, desaparece.

\section{Una propuesta integral}

La Oficina Internacional de la Educación apunta que la estrategia internacional identifica al menos cinco medidas por seguir para enfrentar el problema de la repetición y aumentar el rendimiento académico de los estudiantes: 
a. Expandir la pre-escolaridad: parece haber una correlación positiva entre la educación preescolar y las posibilidades de éxito en el aprendizaje y la reducción de la repetición, especialmente si se asocia con programas de nutrición.

b. Mejorar los métodos de enseñanza de la lecto-escritura y promover la educación bilingüe: para reducir la repetición y mejorar la calidad de la educación básica, debe existir como estrategia, el modificar la modalidad de enseñar y los estilos de apredizaje de la lecto-escritura.

c. Sistemas de evaluación y métodos de enseñanza: la evaluación del aprendizaje suele utilizarse más con fines de selección que de identificación de posteriores dificultades de aprendizaje en el alumno.

En América Latina se han tenido experiencias interesantes de instrucción flexible, es decir, han eliminado los grados y los alumnos progresan a su propio ritmo, incentivan a los más dotados con materiales apropiados y se da ayuda específica a los que tienen mayor problema. Se eliminan las pruebas anuales y se emplea la autoevaluación y el auto-aprendizaje.

d. Mejorar las condiciones del aprendizaje: actualmente en algunos centros educativos, se ofrecen al alumno condiciones de aprendizaje deterioradas, por lo que deben compensarse los déficit que el alumno tiene en la escuela (espacio físico, recursos materiales y textos), con educadores capacitados que puedan -en forma autónoma- adecuar el currículo al medio.

5. Medir mejor el fenómeno de la repetición: debe mejorarse la forma como se recoge la información estadística, así como el análisis de los datos; definiendo operacionalmente los tipos de repetición y las causas que permitan medir el fenómeno, controlar su evaluación y hacer comparaciones, logrando identificar mejor quiénes son los repitentes y ajustar las acciones a estos grupos de población.

Al respecto, Pazos (1991), propone tres líneas de acción tendientes a eliminar el problema del fracaso escolar:

a. Talleres de expresión para los niños más rezagados.

b. Reforzar los vínculos entre las escuelas y las organizaciones comunitarias.

c. Crear espacios de reflexión e intercambio con los maestros más jóvenes y menos conscientes de los problemas que enfrenta este grupo de población.

De la Serna (1995), plantea que lo primero que se debe hacer cuando ya existe el problema, es un psicodiagnóstico para determinar las causas y, en caso necesario, debe darse un tratamiento psicológico o psicopedagógico. 


\section{Mirando hacia el futuro}

Si desde el punto de vista de la escuela tradicional, la repetición, en cierta medida, puede considerarse un procedimiento normal de evaluación y selección, la persistencia de altas tasas de fracaso escolar constituye, en realidad, una contradicción con los objetivos de un sistema educativo que se propone garantizar el aprendizaje de conocimientos básicos a toda la población.

Satisfacer las necesidades básicas de aprendizaje implica varios desafíos y abre nuevas perspectivas para la escuela primaria: en definitiva, debemos asumir el mensaje según el cual todos los niños pueden aprender.

La implementación de estrategias que permitan el diagnóstico temprano de variables afectadas, así como la capacitación del personal docente en el trabajo de áreas problemas deben ser nuestras metas a corto plazo. Paralelo a ello, la promoción de investigaciones de largo alcance que permitan actualizar las causas $y$, por ende, prevenir las consecuencias, debe ser retomada, e implementadas las investigaciones con nuevos recursos e instrumentos de medición exacta.

\section{Referencias}

Alfaro A. Arce P. Solís K. y Vargas L. (1997). Algunos factores psicosociales asociados a la repitencia y la deserción en I y II ciclos. Ministerio de Educación Pública, Departamento de Orientación y Vida Estudiantil. Código 64975 - CIDENAF. Costa Rica.

Brenes, G. (1997). "Entidades psiquiátricas que interfieren en el rendimiento escolar". En: Memoria II Seminario Nacional sobre Rendimiento Escolar: INEINA- CIDE - UNA. Heredia, Costa Rica.

De la Serna, L. (1995). "Fracaso Escolar. ¿De quién es la culpa?, ¿del niño, del maestro o de los padres?”. En: Familia Cristiana. No. XXX. Argentina.

Flores, C. (1991). "La Psicología educativa ante el fracaso escolar". En: Revista Costarricense de Psicología. No. 19, pags. 69 -78. San José, Costa Rica.

Martínez, M. (1997). "Consideraciones Clínicas en torno al Rendimiento Escolar". En: Memoria II Seminario Nacional sobre Rendimiento Escolar. UNACIDE-INEINA 
Massino, A. (1995). "Repetición escolar en la enseñanza primaria: una perspectiva global". En: Revista Argentina de Psicopedagogía. Año 11, No. 38 (2da etapa), invierno 1995. pp. 28 - 42. Buenos Aires, Argentina.

MEP. (2000) Repetición en el sistema educativo costarricense, 1999. San José, Costa Rica

Oficina Internacional de la Educación de la UNESCO. (s.f). "La repetición escolar en la enseñanza primaria: una perspectiva global". En: Novedades Educativas. No. 65. Año 8. Argentina. Pág. 44.

Pazos, C. (1991). " Niños pobres en la escuela. ¿Un fracaso inevitable?". En: Gurises Unidos. Vol 2. No. 4. Agosto p.4 (1) id.

Pernudi, V. (1997). "Rendimiento Académico: habilidades, cognoscitivas y afectivas". En: Memoria Il Seminario Nacional sobre Rendimiento Escolar. UNA-CIDE-INEINA Heredia, Costa Rica.

Rosales, J. (1987). Promoción, repitencia, deserción y desperdicio en educación primaria y secundaria. Centro de Información Computarizada. OEA.

Sautu, R. (1995). “Escolaridad y futuro". En: Oikos: Revista de posgrado, Investigación y Doctorado. Año 3, No. 7 - Abril. Facultad de Ciencias Económicas, Universidad de Buenos Aires, Argentina. 